\title{
LOFAR Transients and the Radio Sky Monitor
}

\author{
Rob Fender ${ }^{*},{ }^{a b}$ Ralph Wijers, ${ }^{b}$ Ben Stappers, ${ }^{b c}$ Robert Braun, ${ }^{c}$ Michael Wise, ${ }^{b c}$ Thijs \\ Coenen, ${ }^{b}$ Heino Falcke, ${ }^{c d}$ Jean-Mathias Griessmeier, ${ }^{e, c}$ Michiel van Haarlem, ${ }^{c}$ Ger \\ de Bruyn,,$^{c}$, Peter Jonker, ${ }^{f g h}$ Casey Law, ${ }^{b}$ Sera Markoff, ${ }^{b}$ Joseph Masters, ${ }^{b}$ James \\ Miller-Jones, ${ }^{b}$ Rachel Osten, ${ }^{i}$ Bart Scheers, ${ }^{b}$ Hanno Spreeuw, ${ }^{b}$ John Swinbank, ${ }^{b}$ \\ Corina Vogt, ${ }^{c}$ Rudy Wijnands ${ }^{b}$ and Philippe Zarka, ${ }^{e}$
}

${ }^{a}$ School of Physics and Astronomy, University of Southampton, Highfield, Southampton, SO17 $1 B J, U K$

${ }^{b}$ Astronomical Institute 'Anton Pannekoek', University of Amsterdam, Kruislaan 403, 1098 SJ, Amsterdam, the Netherlands

' Stichting ASTRON, Postbus 2, 7990 AA Dwingeloo, the Netherlands

${ }^{d}$ Department of Astronomy, Radboud University, Postbus 9010, 6500 GL Nijmegen, the Netherlands

e Observatoire de Paris-Meudon, 5 Place Jules Janssen, 92195 Meudon Cedex, France

${ }^{f}$ SRON, Netherlands Institute for Space Research, Sorbonnelaan 2, 3584 CA, Utrecht, the Netherlands

${ }^{g}$ Harvard-Smithsonian Center for Astrophysics, 60 Garden Street, Cambridge, MA 02138, USA

${ }^{h}$ Astronomical Institute, Utrect University, Postbus 80000, 3508 TA, Utrecht, the Netherlands

${ }^{i}$ Department of Astronomy, University of Maryland, College Park, MD, USA

E-mail: rpfephys.soton.ac.uk, rwijers@science.uva.nl,ben. stappers@manchester.ac.uk, robert.braun@csiro.au, wise@science.uva.nl, tcoenen@science.uva.nl, falcke@astron.nl, griessmeierdastron.nl, haarlem@astron.nl, p.jonker@sron.nl, claw@science.uva.nl, sera@science.uva.nl, jmastersescience.uva.nl, jmiller@science.uva.nl, rosten@astro.umd.edu, bscheers@science.uva.nl, hspreeuw@science.uva.nl, swinbank@science.uva.nl vogteastron.nl, rudy@science.uva.nl, philippe.zarka@obspm.fr

The study of transient and variable low-frequency radio sources is a key goal for LOFAR, with an extremely broad science case ranging from relativistic jets sources to pulsars, exoplanets, radio bursts at cosmological distances, the identification of gravitational wave sources and even SETI. In this paper we will very briefly summarize the science of the LOFAR Transients key science project, will outline the capabilities of LOFAR for transient studies, and introduce the LOFAR Radio Sky Monitor, a proposed mode in which LOFAR regularly scans $\sim 2 \pi$ radians of sky.

\footnotetext{
*Speaker.
} 


\section{Introduction}

LOFAR is a next-generation radio telescope under construction in The Netherlands with longbaseline stations under development in other European countries (currently Germany, The UK, France, Sweden). The array will operate in the $30-80$ and $120-240 \mathrm{MHz}$ bands $(80-120 \mathrm{MHz}$ being dominated by FM radio transmissions in northern Europe). The telescope is the flagship project for ASTRON, and is the largest of the pathfinders for the lowest-frequency component of the Square Kilometere Array (SKA). Core Station One (CS1; see Gunst et al. 2006) is currently operating, and the next stage of deployment is about to begin, with 36 stations to be in the field by the end of 2009.

For more information on the project, see

\section{http://www.lofar.org}

The science of LOFAR has been driven intially by four, and more recently six, Key Science Projects (KSPs). These are:

- The Epoch of Reionization: LOFAR will detect the signal of the $21 \mathrm{~cm} \mathrm{HI}$ line redshifted to the era, a billion years after the big bang, when the first sources of radiation (Pop III stars and the first accreting black holes) began to reionize the Universe.

- Extragalactic Surveys: LOFAR will conduct the most extensive surveys of extragalactic radio sources (AGN and starburst galaxies) ever undertaken, not to be surpassed until the completion of the SKA.

- Transients and pulsars: LOFAR will revolutionize the study of bursting and transient radio phenomena, including pulsars, due to its enormous field of view and the very low frequencies which maximise sensitivity to coherent radio bursts.

- Cosmic rays: LOFAR will detect geosynchrotron radiation from cosmic ray air showers, and will attempt to detect the highest particles energies ever measured by looking for their interaction with the lunar regolith.

- Solar physics: LOFAR will study the explosive particle acceleration in the Sun's atmosphere, and the propagation of ejecta through the inner solar system.

- Cosmic Magnetism: LOFAR will measure the ordering of magnetic fields on all scales from our own galactic plane to galaxy clusters, via deep measurements of polarisation.

In addition to these KSPs, there will be an increasing fraction of open time available to the wider user community. In this brief paper, we highlight the science and implementation of the Transients KSP, and in particular the very wide-field scan mode which we call the Radio Sky Monitor (RSM).

\section{The LOFAR Transients Key Science Project}

The LOFAR Transients Key Science Project (KSP) aims to study all variable and transients sources detected by LOFAR. Such sources can be broadly divided into two types: 
1. Incoherent synchrotron emission: essentially all explosive events which inject energy into the ambient medium result in particle acceleration and/or compression/enhancement of magnetic fields, resulting in synchrotron emission. Such emission is likely to be initially selfabsorbed at LOFAR frequencies, with a rise time corresponding to the timescale for the source to expand and become optically thin in a given band. This expansion timescale is proportional to the initial size of the emitting region divided by the expansion velocity. In approximate order of increasing rise time, sources which will be associated with such emission are jets from CVs, X-ray binaries, GRBs and SNe, and finally AGN. Under most conditions, synchrotron emission has a maximum brightness temperature of $\sim 10^{12} \mathrm{~K}$. An example of an outburst from a galactic binary system is given in Fig 1 below.
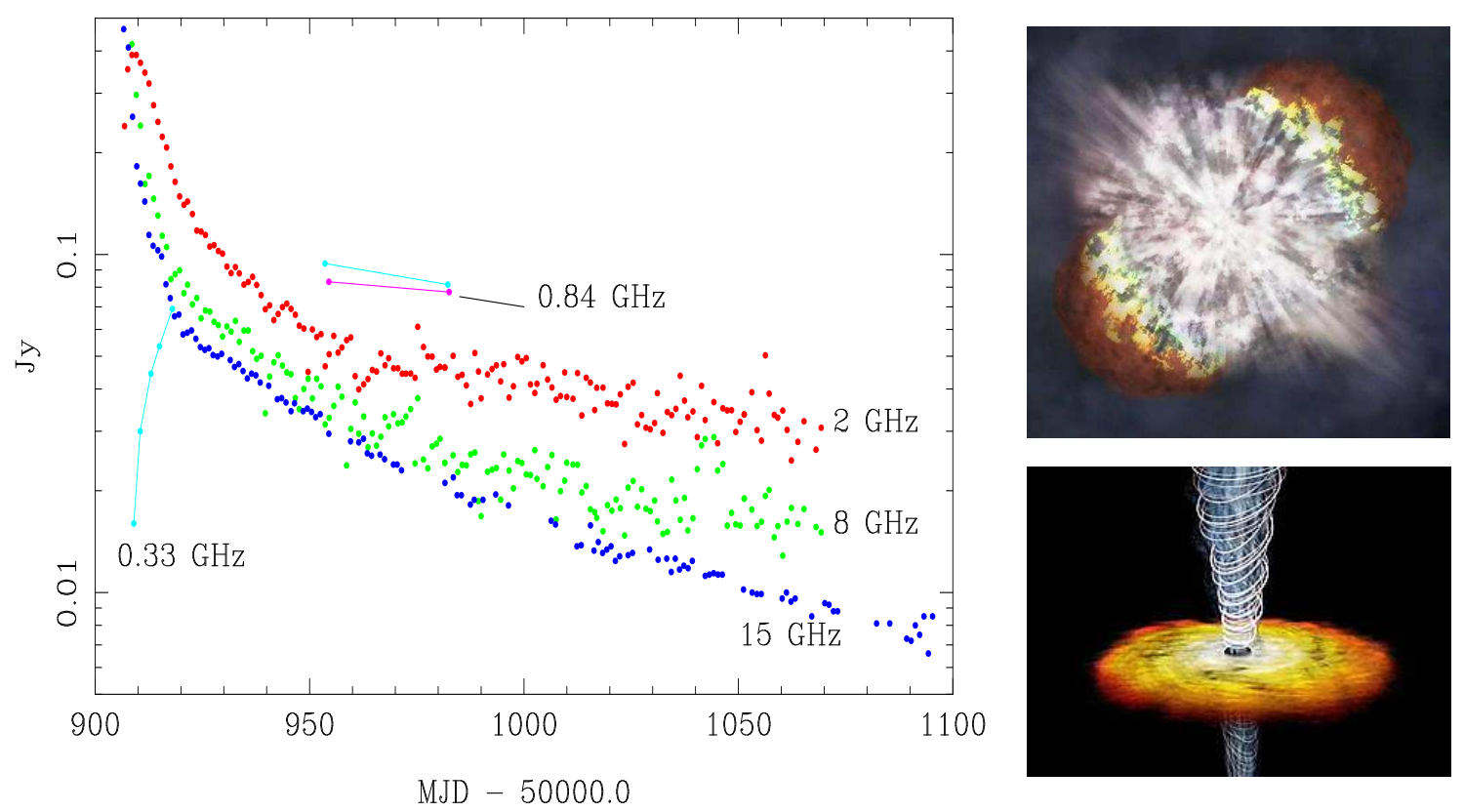

Figure 1: Evolution of a radio outburst from the X-ray transient CI Cam, with data from the Ryle Telescope $(15 \mathrm{GHz})$, the Green Bank Interferometer $(2$ and $8 \mathrm{GHz})$ and the Westerbork Synthesis Radio Telescope $(0.8$ and $0.3 \mathrm{GHz}$ ). The radio signal is due to synchrotron emission from an expanding source of accelerated electrons. By the time of the first radio observations ('externally triggered' by X-ray observations) the radio source is already optically thin at the highest frequency, $15 \mathrm{GHz}$. As the source expands, the flux density at different frequencies increases as the optical depth at that frequency decreases, but then subsequently decays once in the optically thin regime as expansion results in energy losses. The emission at $330 \mathrm{MHz}$, just above the LOFAR band, was detectable within a few days of the outburst, but did not peak until 20-30 days later. Such behaviour will be characteristic of explosive outburst events associated with e.g. relativistic jets, supernovae, GRB afterglows (as indicated by the cartoons to the right of the figure), with the rise and decay timescales being an increasing function of the luminosity of the event.

Note that many of the types of object responsible for explosive particle acceleration, e.g. X-ray binaries, Gamma-ray bursts, are of intense interest to the high-energy astrophysics. LOFAR detections of such events, especially with the Radio Sky Monitor (see below), will undoubtedly therefore be used as triggers for optical / infrared / X-ray / gamma-ray followup (note for a typical X-ray binary outburst, the majority of the interesting X-ray behavior 
would still be observed even if the LOFAR trigger was $\sim 20$ days after the initial outburst due to the optical depth delay described above). Taken as an ensemble, observations of the synchrotron sources will provide a complete time-resolved census of particle acceleration in the local universe, shedding light on the energization of ambient media and sites of cosmic ray acceleration.

2. Coherent emission: several different types of radio emission with brightness temperatures $\geq 10^{12} \mathrm{~K}$, often resulting from very short durations, are lumped together under the title of 'coherent' radio emission. This is generally taken to mean groups of electrons moving together en masse, and may often be highly anisotropic (e.g. maser emission). Several different classes of coherent radio emitters are likely to be detected by LOFAR, e.g.

- Flare stars, brown dwarfs and active binaries are likely to be present in almost every LOFAR beam, giving off highly circularly-polarised radio bursts from coherent emission processes. Potential targets include $\mathrm{M}$ dwarf flare stars, active binaries like RS CVn and low mass $\mathrm{L}$ and $\mathrm{T}$ dwarfs.

- LOFAR will also study radio emission from planets both within and beyond the Solar System. This includes imaging Jupiter's magnetosphere at high spatial and time resolution, imaging Jupiter's radiation belts, and studying planetary lightning from the other planets within the Solar System. It is also predicted that radio bursts from nearby so-called 'hot Jupiter' exoplanets might also be detected, and we will carry out a detailed survey for such objects. If successful, we would have an inclination independent catalogue of extrasolar planets, including possible diagnostics of their magnetic fields and rotation periods.

- LOFAR may detect extragalactic radio bursts, such as that reported by Lorimer et al. (2007; Fig 2), to very large distances, possibly as far as $z \sim 7$, providing a unique probe of the properties of the intergalactic medium (via their dispersion measure). Such events may be even be associated with neutron star-neutron star mergers, in which case the radio identification of events detectable by advanced LIGO may be possible. Since such mergers are predicted to provide independent distance measurements based on their gravitational wave signatures, identification of the electromagnetic counterpart would provide a unique test of gravity on cosmological scales, as well as an independent test of the redshift-distance relation.

In addition to all this, a major survey of classical radio pulsars will be undertaken, as well as the study of related objects such as Anomalous X-ray Pulsars (AXPs) and Rotating Radio Transients (RRATs). The LOFAR pulsar survey is expected to discover more than 1000 new pulsars (see Fig 3), which will provide the majority of pulsars for the Pulsar Timing Array (Foster \& Backer 1990) in the northern hemisphere. Such a survey also has a fair chance of turning up the first pulsar - black hole binary. In addition, LOFAR will provide the sensitivity to allow us to study the individual pulses from an unprecedented number of pulsars including millisecond pulsars and the bandwidth and frequency agility to study them over a wide range which will provide vital new input for models of pulsar emission. This will provide us with an unparalleled survey of the population of massive star end-products within our galaxy. 


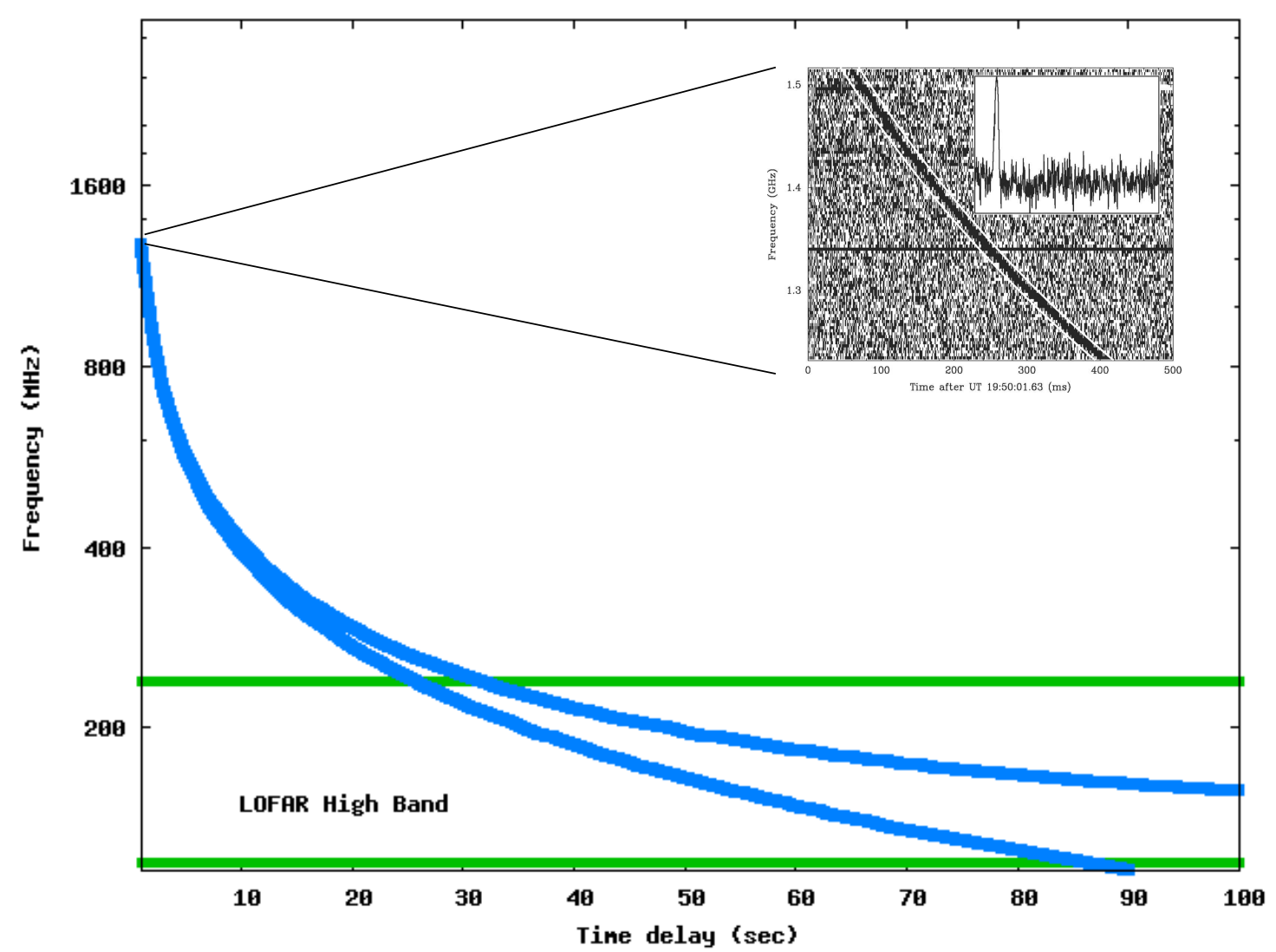

Figure 2: An illustration of how the highly-dispersed extragalactic radio burst reported by Lorimer et al. (2007) would sweep through the LOFAR high band (the frequency limits of which are indicated by the green horizontal lines) some tens of seconds later. The blue lines indicate the estimated delay and width of the pulse. The dispersion delay is assumed to be quadratic; the scatter-broadening of the signal is assumed to grow as $v^{-4}$ and we assume a pulse width of $5 \mathrm{~ms}$ at $1.4 \mathrm{GHz}$ (note that the pulse was not resolved by Lorimer et al. and so this may be considered to be an upper limit to the scattered pulse width). If the measured steep spectrum $\left(S_{v} \propto v^{-4}\right.$, the same as the scatter broadening) extends to low frequencies, such a burst would be detectable in the LOFAR standard data products up to distances in excess of a Gpc, allowing unprecedented studies of dispersion and scattering in the IGM. Such an event may even have been associated with a neutron star-neutron star merger. If so, such events may be detectable by LIGO, and a distance inferred from the gravitational wave signal alone. LOFAR identification of the host galaxy, via precise localisation of the burst, would allow two independent measurements of distance on cosmological scales, providing a unique test of gravity and of the distance-redshift relation.

Finally, beyond all this, LOFAR is almost guaranteed to produce many exciting physical discoveries simply because of the enormous range of parameter space it is exploring. This could include SETI, for which, depending on the nature of the signal, LOFAR may be the best hope prior to the SKA. This discovery space, and the distance to which LOFAR may detect different phenomena with different timescales, is illustrated in Fig 4.

More information on the Transients KSP can be found at

http://www.astro.uva.nl/lofar_transients/ 

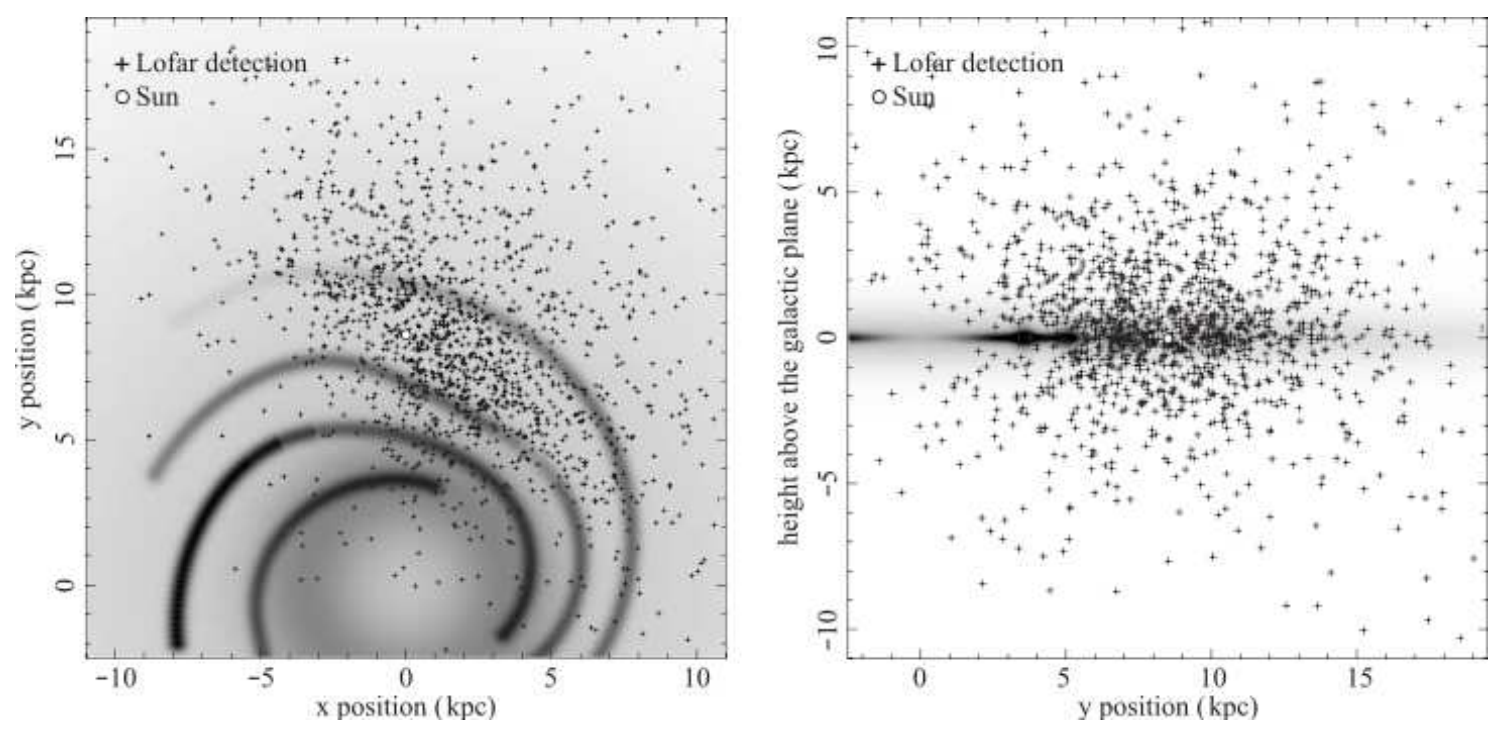

Figure 3: The 1000+ pulsars discovered in a 60-day LOFAR all-sky survey simulation. ISM shown in gray. Left) projected on the Galactic plane. Right) projected on the plane through the Galactic centre and sun, perpendicular to the disk. From van Leeuwen \& Stappers (2008).

\section{The Radio Sky Monitor}

The Transients KSP will study variable radio sources via four distinct approaches:

1. We will rapidly and regularly scan a large fraction of the entire northern sky in the Radio Sky Monitor mode. This mode is the subject of the majority of the rest of this paper.

2. We will undertake surveys in a phased-array mode, allowing e.g. high time resolution exoplanet / pulsar searches.

3. In piggyback mode we will attempt to seach all LOFAR observations to look for variable and transient sources, by comparing with previous images of that region of sky.

4. We will also perform targeted deep / high-resolution observations of specific key astrophysical sources, often in concert with other facilities - e.g. orbiting X-ray observatories, groundbased optical telescopes.

\subsection{LOFAR configurations}

LOFAR will employ stations of differing sizes depending on their location:

- Core In the LOFAR core, High-Band Antennae (HBAs) will be grouped into sub-stations of 24 tiles, two such sub-stations associated with one 48-dipole Low-Band Antenna (LBA) station (96 LBAs are actually deploted, but only 48 can be selected at any given time).

- Intermediate baselines On intermediate baselines (i.e. those within The Netherlands, outside of the core, LOFAR stations will be comprised of one 48-tile set of HBAs and one 48-dipole LBA station (actually 48 of 96, as in Core).. 


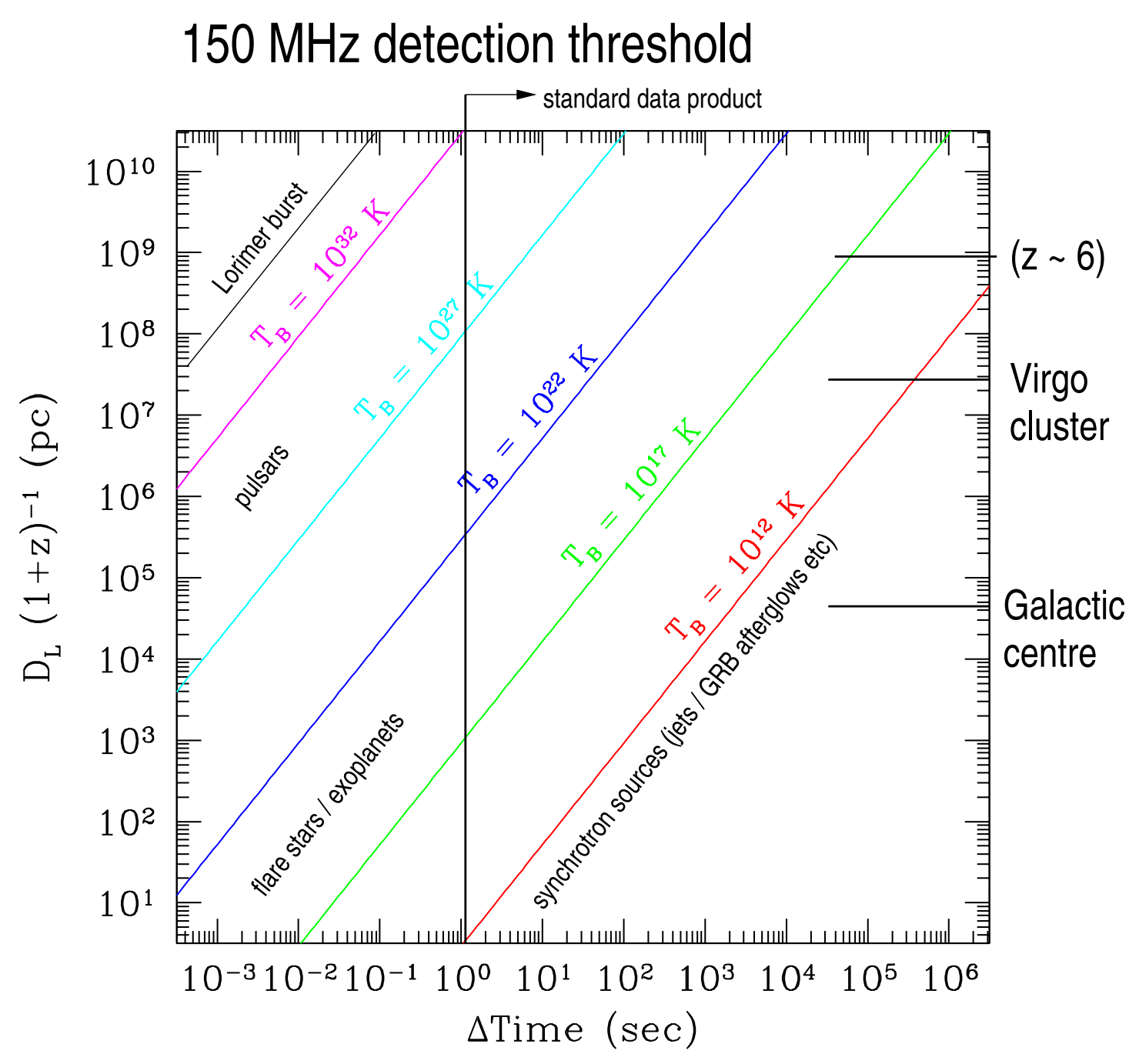

Figure 4: An illustration of discovery space possibilities for LOFAR when searching for variable and transient phenomena. The diagonal lines delineate the upper bound to the region below which detection is possible at a given distance and on a given timescale. Also indicated are the distances of the galactic centre, Virgo cluster and a redshift of $z \sim 6$. Variable Synchrotron sources at all distances will be detectable with the standard LOFAR data products, which are maps every second. Higher brightness temperature phenomena such as flare stars and, we predict, exoplanets are likely to show temporal structure on much shorter timescales and so in order to obtain the most astrophysical information higher time resolution modes will be required. Very high brightness temperature phenomena such as pulsars or the 'Lorimer transient' (Lorimer et al. 2007) could be detected at enormous distances and in many cases will be dispersed over timescales long enough to allow their identification in standard data products (see e.g. Fig 2). The detection thresholds are estimated for a $32 \mathrm{MHz}$ bandwidth. 


\begin{tabular}{|c|c|c|c|c|c|c|c|c|c|}
\hline \multirow[t]{2}{*}{ Configuration } & \multirow[t]{2}{*}{$\mathrm{N}$} & \multirow{2}{*}{$\begin{array}{l}b_{\max } \\
(\mathrm{km})\end{array}$} & \multirow{2}{*}{$\begin{array}{c}v \\
(\mathrm{MHz})\end{array}$} & \multicolumn{2}{|c|}{ HPBW } & \multirow[t]{2}{*}{$N_{2 \pi}$} & \multirow{2}{*}{$\begin{array}{c}\theta \\
(\operatorname{arcsec})\end{array}$} & \multirow{2}{*}{$\begin{array}{c}\sigma_{1 \mathrm{hr}} \\
(\mathrm{mJy})\end{array}$} & \multirow{2}{*}{$\begin{array}{c}\sigma_{2 \pi} \\
(\mathrm{mJy})\end{array}$} \\
\hline & & & & (deg) & (sr) & & & & \\
\hline \multirow{2}{*}{ Core } & \multirow[t]{2}{*}{$768+$} & \multirow[t]{2}{*}{2} & 30 & 9.1 & 0.079 & 160 & 1345 & 20 & 50 \\
\hline & & & 120 & 5.3 & 0.027 & 470 & 330 & 0.6 & 2.2 \\
\hline \multirow{2}{*}{ LOFAR-NL } & \multirow{2}{*}{$1536+$} & \multirow[t]{2}{*}{100} & 30 & 9.1 & 0.079 & 160 & 25 & 10 & 25 \\
\hline & & & 120 & 3.7 & 0.013 & 960 & 7 & 0.3 & 1.9 \\
\hline \multirow{2}{*}{ E-LOFAR } & \multirow[t]{2}{*}{$2000+$} & \multirow[t]{2}{*}{1000} & 30 & 11.5 & 0.126 & 100 & 1 & 6.5 & 13 \\
\hline & & & 120 & 2.9 & 0.008 & 1560 & 0.4 & 0.2 & 1.6 \\
\hline
\end{tabular}

Table 1: $\mathrm{N}$ is total number of HBA tiles or LBA dipoles, $v$ is observing frequency, HPBW is the half power beam width (三 FWHM) in both degrees and steradians. $N_{2 \pi}=(1-\cos (\mathrm{HPBW} / \sqrt{2}))^{-1}$ is the number of beams required to tile out one entire hemisphere, assuming an offset between pointing centres of $($ FWHM $) / \sqrt{2}$ as used by Condon et al. (1998) in the NVSS (see Fig 5 below for example of this configuration for LOFAR beams). The angular resolution is $\theta$ and $\sigma_{1 \mathrm{hr}}$ is the sensitivity (rms) in one hour. The sensitivity of a uniform hemispherical survey, conducted over $24 \mathrm{hr}$, is given by $\sigma_{2 \pi}$. A $4 \mathrm{MHz}$ bandwidth is assumed. The sensitivity scales with observation length as $\sqrt{t}$, similarly with bandwidth as $\sqrt{\text { bandwidth and }}$ linearly with the number of antennae $N$. Note that the field of view varies depending on the station layout which dominates in each configuration.

- International baselines On the longest (international) baselines, LOFAR stations will consist of 96 HBAs and 96 LBAs

The inhomogenous design outlined above allows LOFAR to optimise both the imaging quality and survey speed of the telescope for a fixed budget. Within The Netherlands, it is anticipated that the Core will correspond to 16 stations (i.e. 768 HBA tiles and 768 LBA dipoles) within a diameter of $\sim 2 \mathrm{~km}$.

\subsection{Observations}

The effective collecting area and field of view of LOFAR peaks at the lower bound of the two discrete observing bands: $30 \mathrm{MHz}$ for the LBAs and $120 \mathrm{MHz}$ for the HBA tiles. It is at these two frequencies that the LOFAR RSM will operate (with possible follow-ups at higher frequencies).

Specifically, at $30 \mathrm{MHz}$ LOFAR offers the widest possible field of view. This is also likely to be the frequency at which coherent events (which constitute much of the hoped-for exploration of new parameter space) are strongest. However, spectral index is a key diagnostic of the nature of a newly-identified source, and a rapid classification of such events is a key goal of the TKP. In addition, 'synchrotron bubble' events (associated with e.g. GRB afterglows and some X-ray binary outburst) will peak earlier and stronger at higher frequencies. In addition, the higher frequency will allow better localisation of sources, and the HBAs at $120 \mathrm{MHz}$ are more sensitive than the LBAs at $30 \mathrm{MHz}$ (the factor $\sim 30$ difference in $\sigma$ would be nullified by a spectral index steeper than $\alpha \sim-2.5)$. Finally, dispersion and pulse broadening are strong functions of frequency, and will be far less severe in the high band than the low. We therefore propose to operate the RSM at both 30 $\mathrm{MHz}$ and $120 \mathrm{MHz}$.

Using seven beams to tile out a hexagonal pattern (see Fig 5), operating the LOFAR Radio Sky Monitor from the core (ie. with maximum baselines of $\sim 2 \mathrm{~km}$ ) will result in an instantaneous 


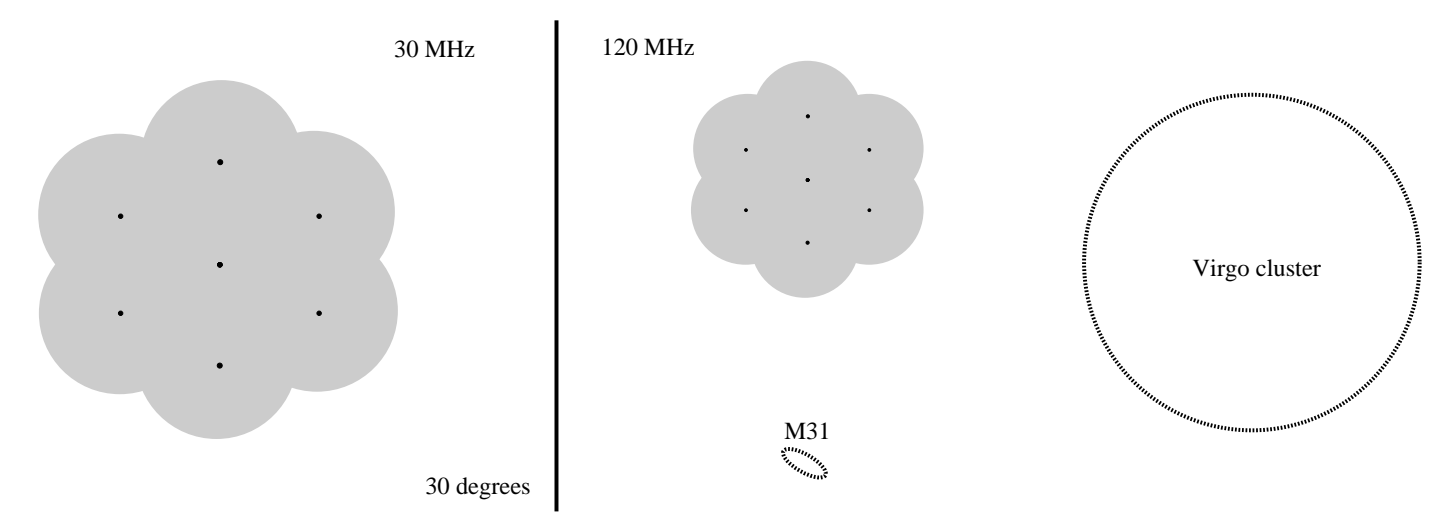

Northern galactic plane

Figure 5: An example of a Radio Sky Monitor (RSM) observing modes. In this example a hexagonal pattern is traced out using seven beams and an offset between pointing centres of FWHM $/ \sqrt{2}=6.4$ and 3.7 degrees at 30 and $120 \mathrm{MHz}$ respectively. The approximate angular size on the sky of M31, the Virgo cluster and the northern galactic plane are indicated for comparison. The angular resolution within these fields of view depends upon the longest baselines used - see Table 1 .

field of view of $\sim 0.08$ steradians at $120 \mathrm{MHz}$ and $\sim 0.2$ steradians at $30 \mathrm{MHz}$. This hexagonal pattern, with an offset between pointings of $(\mathrm{FWHM})^{0.5}=6.4$ and 3.7 degrees at 30 and 120 $\mathrm{MHz}$ respectively, gives the most uniform sky coverage for wide field surveys (e.g. Condon et al. 1998 for the NRAO VLA sky survey). These fields of view are extremely large and illustrate the unprecedented monitoring and surveying capabilities of the telescope.

A variety of strategies for operating the RSM mode can be envisaged, e.g.

- Rapid All-Sky Monitoring: Rapid shallow half-sky (hemispherical) surveys could be performed on short timescales in order to survey for rapid transients. In table $1, N_{2 \pi}$ corresponds to the number of beams required to tile out an entire hemisphere on the sky. This varies from of order 100 at $30 \mathrm{MHz}$ to $\sim 1600$ for $120 \mathrm{MHz}$ with international stations (which are larger and therefore have a smaller field of view). This is a very small number of pointings in order to survey half of the entire sky, and means that several minutes could be spent at each point in the sky (if such a scan were carried out over something like one day). This in turn means that $\sim$ mJy sensitivity (see $\sigma_{2 \pi}$ in table 1 ) surveys can be carried out daily.

- Zenith monitoring: Staring at the zenith optimises the sensitivity and beam stability of the telescope, whilst providing a sizeable and repeatedly monitored part of the sky. Using the patterns illustrated in Fig 5, mapping out the entire field of view which passes the zenith can be achieved in approximately 20 pointings at $30 \mathrm{MHz}$ and 30 at $120 \mathrm{MHz}$, tracking each field for about an hour, achieving (sub-)mJy sensitivity. In RA, Dec terms this delineates a strip at $54^{\circ}$ north of full width 26 degrees (i.e. extending from Dec +41 to +67 ).

- Galactic plane monitoring: Most of the northern galactic plane is visible from LOFAR and could be monitored for galactic transients. A large fraction of the 'known' radio transients, 


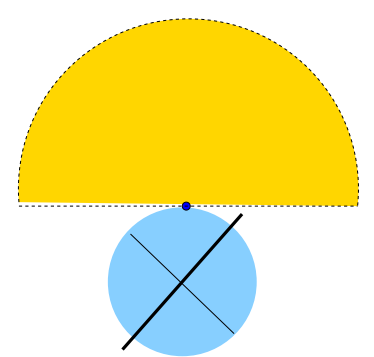

Hemispherical survey

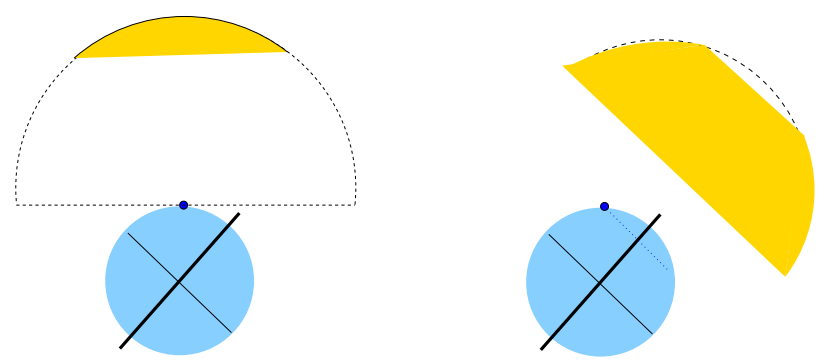

Zenith monitoring program

Figure 6: Possible modes for observing with the LOFAR Radio Sky Monitor (RSM). Rapid all-hemisphere surveys are possible (see $N_{2 \pi}$ and $\sigma_{2 \pi}$ in Table 1. Alternatively, we may stare at the zenith, where the array is most sensitive and best calibrated, and allow rotation of the Earth to tile out a significant fraction of the sky.

such as those associated with rotation-powered neutron stars and X-ray binary systems, are heavily concentrated towards the galactic plane (note that many sources e.g. flare stars, GRB afterglows, AGN are not). The RSM zenith-monitoring mode will sample the galactic plane between about $90 \leq l \leq 160$. However, much of the galactic plane will not be well sampled by this program, especially towards the galactic centre. In order to counterbalance this, we propose regular scans of the galactic plane. Since the galactic plane will also, however, suffer most from strong field sources and heavy dispersion at $30 \mathrm{MHz}$, these surveys will only be conducted at $120 \mathrm{MHz}$.

Fig 6 sketches out the hemispherical and zenith-monitoring modes.

\section{Analysis}

Our aim is that the data will be correlated, calibrated and delivered to Amsterdam at a rate of one image per beam per second, corresponding to the LOFAR 'standard data products'. Delivery of maps made from longer, logarithmically-spaced time intervals, from the central computing cluster, will result in a total image transfer rate of approximately two per beam per second, i.e. 48 images / second for the RSM. Images will be analysed in real time (via source-detection software currently under development by the TKP) to check both for new transients and for the fluxes of known objects. Of course non-standard modes, such as phase-arrays for pulsar and exoplanet searches, will require different routes to transient discovery and classification.

Any new transients will have their data fed into a classification / alert pipeline which may under certain circumstances trigger follow up observations (such as full-array and frequencies up to $240 \mathrm{MHz}$ ). A sketch of how such a pipeline may integrate with the LOFAR central processing is presented in Fig 7. It will be our policy that alerts will passed to the broader community as well as directly to partner observatories. The fluxes of known variable sources will be fed into the transients database (currently in the design phase) and may also trigger follow-up observations if there are particularly dramatic changes. 


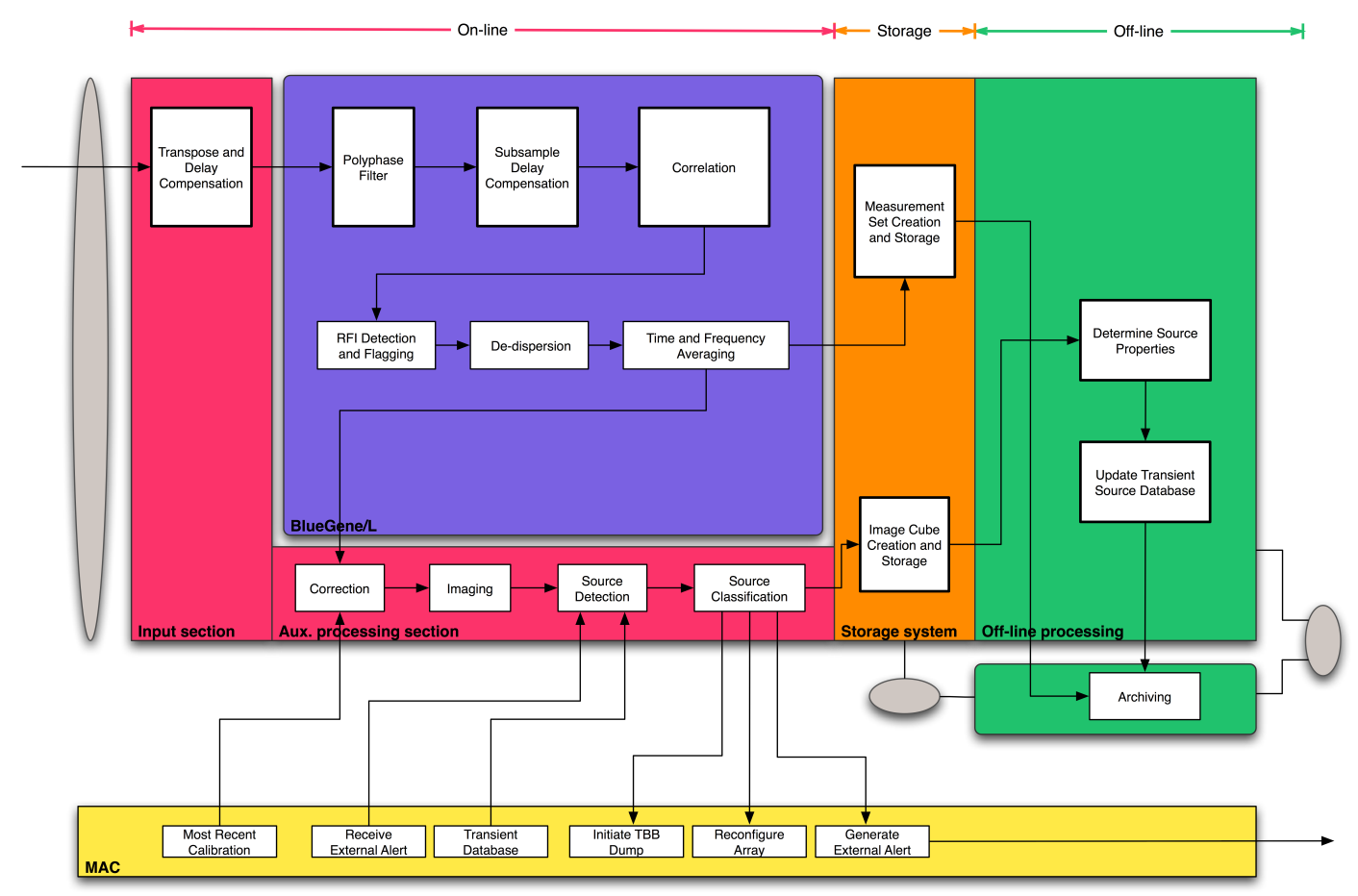

Figure 7: An simple illustration of how transients pipelines are to be integrated into the processing system for LOFAR.

\section{References}

[1] Condon J.J., Cotton W.D., Greisen E.W., Yin Q.F., Perley R.A., Taylor G.B., Broderick J.J., 1998, AJ, 115,1693

[2] Foster R.S., Backer D.C., 1990, ApJ, 361, 300

[3] Gunst A., van der Schaaf K., Bentum M.J., published in the Proceedings from SPS-DARTS 2006, The second annual IEEE BENELUX/DSP Valley Signal Processing Symposium, March 28-29 (2006), Antwerp, Belgium

[4] Lorimer D.R., Bailes M., McLaughlin M.A., Narkevic D.J., Crawford F., 2007, Science, 318, 777

[5] van Leeuwen J., \& Stappers B., 2008, published in 40 YEARS OF PULSARS: Millisecond Pulsars, Magnetars and More. AIP Conference Proceedings, Volume 983, pp. 598-600 (2008)

(arXiv:0710.0675) 